\title{
Intestinal Microbiota Transplantation: A Case of Crohn's Colitis with Superimposed Clostridium difficile Infection
}

S Smith

\begin{abstract}
INTRODUCTION
Clostridium difficile infection when associated with inflammatory bowel disease (IBD) carries a higher mortality than in the absence of underlying IBD (1). While the use of antibiotics is a well-known risk factor for this infection, so too are the use of proton pump inhibitors, in the peri-partum and postoperative periods (4). The incidence of $C$ difficile infection has more than doubled in the United States of America (USA) since 1996 (5). Unresponsiveness to treatment as well as disease recurrence occur with equal frequency following conventional treatment with metronidazole and oral vancomycin. Twenty to thirty per cent of patients relapse after being treated with either of these agents (6). A recent systematic review of the use of fecal bacteriotherapy in patients with recurrent disease revealed that this form of treatment is associated with a $92 \%$ cure rate.
\end{abstract}

Keywords: Caribbean, Clostridium difficile infection, Crohn's colitis, fecal bacteriotherapy, fecal transplantation

\section{CASE REPORT}

A 35-year old Trinidadian woman had been well up until nine weeks prior to being first seen. She had used misoprostol (cytotec) to induce an abortion, and following this, underwent a dilatation and curettage at a private health facility. After the procedure, her doctor prescribed amoxicillin and clarithromycin for one week. She took them for three days only as she began experiencing abdominal cramps and diarrhoea. She visited a second doctor and received a further prescription for metronidazole, which she again took for just two days. She related that she had been having roughly seven or eight watery, blood-stained stools during the day, and was awakened at night by four or five similar episodes. There was fecal urgency associated with explosive evacuation that produced mostly frank blood. She was started on mesalazine $4.8 \mathrm{gm} /$ day and folic acid, and a colonoscopy scheduled.

Her colon exhibited extensive confluent colitis characterized by exophytic inflammatory change which progressively worsened proximal to the rectum, and extended to the mid-transverse colon (Fig.1). The colonoscopy was not extended beyond that point as the intense inflammation made the procedure very painful. Histology on mucosal specimens

From: San Fernando General Hospital, 20 Herrera Street, St Joseph village, San Fernando, Trinidad and Tobago.

Correspondence: Dr S Smith, San Fernando General Hospital, 20 Herrera Street, St Joseph village, San Fernando, Trinidad and Tobago. E-mail: icteruss@yahoo.com

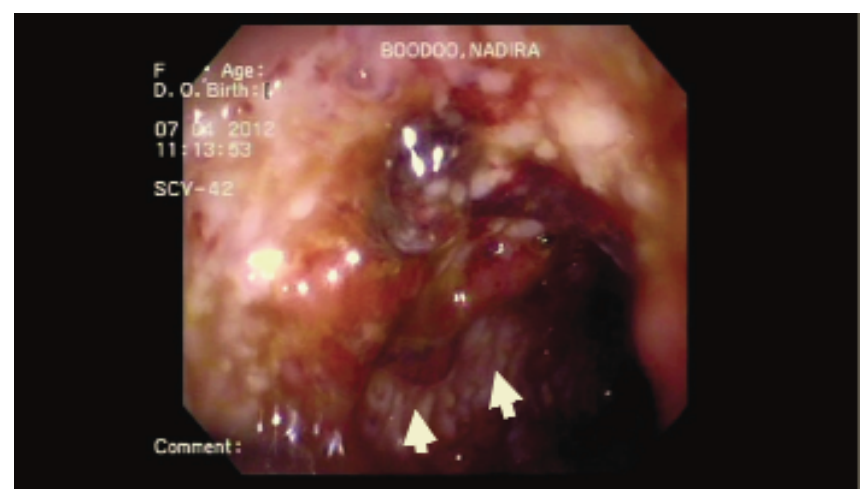

Fig. 1: The typical pseudomembranes seen in $C$ difficile colitis (arrowheads) along with exophytic inflammatory change in the proximal descending colon. Areas of ulceration and spontaneous bleeding are also shown.

reportedly showed mucosal and sub-mucosal lymphocytic infiltrates with fissuring consistent with Crohn's. The patient was started on oral prednisolone $45 \mathrm{mg}$ daily, along with the mesalazine ( $4.8 \mathrm{gm} /$ day) and was asked to return in five days. At that time, she was found to be dehydrated and tachycardic, with a sitting blood pressure (BP) of 105/60 $\mathrm{mmHg}$ and a heart rate (HR) of 120/minute. She was mildly febrile and looked ill. Her mucous membranes were pale. Her abdomen was diffusely tender, more so in the suprapubic region; there was no rebound. She was hospitalized, intravenous fluids started and parenteral analgesics (pethidine) given. She was started on a high calorie liquid elemental diet and parenteral hydrocortisone; oral mesalazine was continued. On days one and two of that admission, stools were sent for $C$ difficile toxin A and B and clostridial antigen [C DIFF QUIK CHEK COMPLETE - TECHLAB ${ }^{\mathrm{TM}}$, Blacksburg, VA, USA]. Both specimens were reportedly strongly positive for toxin A and $\mathrm{B}$ and the clostridial antigen. The patient was started on oral vancomycin - with an initial dose of $125 \mathrm{mg}$ every six hours, increasing to $500 \mathrm{mg}$ every six hours after one week - and parenteral metronidazole $500 \mathrm{mg}$ every eight hours. Plain abdominal X-ray at that time showed no evidence of colonic dilatation (Fig. 2). Over the ensuing two days, her diarrhoea lessened to four episodes of blood-stained stools per day. She was discharged on oral metronidazole $400 \mathrm{mg}$ three times daily and vancomycin $500 \mathrm{mg}$ every six hours, along with mesalazine and prednisolone, with scheduled follow-up in five days. A diagnosis of Crohn's colitis complicated by $C$ difficile infection was made.

When reviewed at follow-up, there was no improvement in her condition in spite of her week of continuous treatment. The diarrhoea and bleeding had worsened and she looked toxic. She was readmitted and fluid resuscitation 


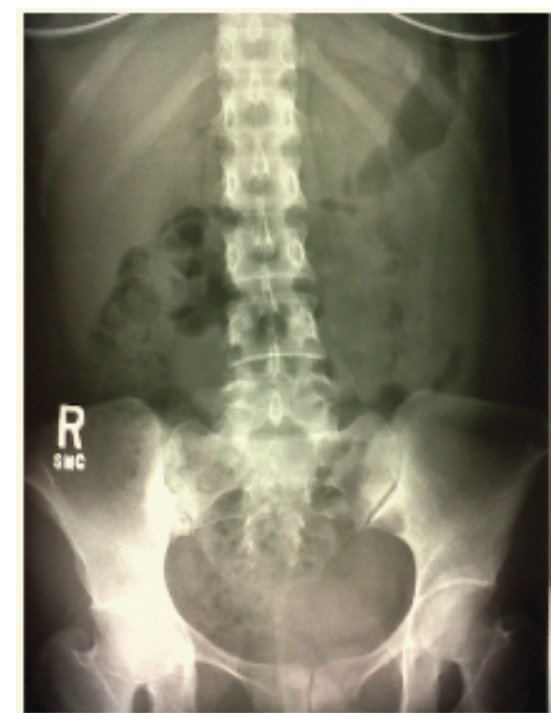

Fig. 2: A plain erect $X$-ray of the patient's abdomen at initial presentation. It shows no colonic dilation.

commenced. Over the ensuing five days, her haemoglobin fell from $10.3 \mathrm{~g} / \mathrm{dL}$ to $7.1 \mathrm{~g} / \mathrm{dL}$ with a leukocyte count of 15.2 $\mathrm{K} / \mu \mathrm{L}$ (87.4\% neutrophils). Her serum albumin fell from 2.7 $\mathrm{g} / \mathrm{dL}$ to $1.8 \mathrm{~g} / \mathrm{dL}$. Two units of whole blood were transfused and both vancomycin and metronidazole were continued. She had developed a toxic megacolon (Fig. 3A-B); the

A

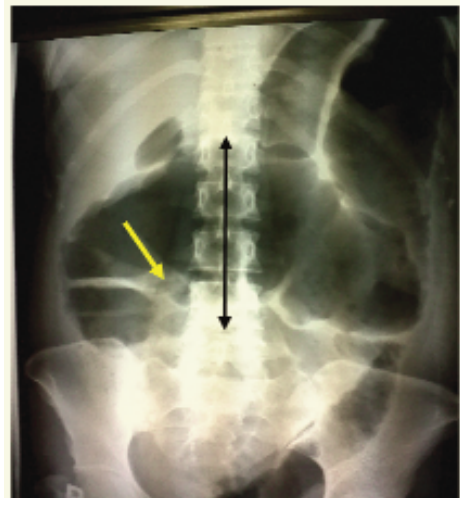

B

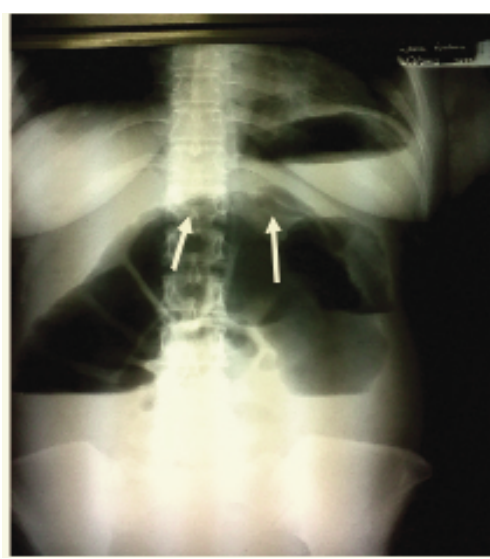

Fig. 3A-B: Maximum diameter of the colon $>8 \mathrm{~cm}$ (A). There was significant mucosal thickening with an appearance approaching 'thumbprinting' [arrows] (B). maximal diameter of the colon exceeded $8 \mathrm{~cm}$ on a plain erect film. The mesalazine was stopped since this drug is known to cause diarrhoea occasionally; however, this did not change the situation.

At this point, it was felt that two treatment options were available: first, intestinal microbiota transplantation - a viable option as both Crohn's and pseudomembranous colitis had been reportedly successfully treated in this way (8). The second plausible option was an emergency sub-total colectomy as a life-saving procedure. These were carefully explained to both the patient and her husband and informed consent was obtained for the first. Her husband had been previously fully immunized against Hepatitis B and was HIV negative; urgent investigations of his stools for toxin $\mathrm{A} / \mathrm{B}$, for pathogens on culture, and for ova and parasites on microscopy were all reportedly negative.

Her husband supplied a healthy stool specimen - the product of a full bowel evacuation produced less than three hours previously. The specimen was prepared and delivered into the recipient colon via the colonoscope in accordance with accepted practice (9). In the initial four hours following the procedure, the patient's condition continued to deteriorate. She became tachycardic - HR 140/minute - her oxygen saturation in ambient air fell to $80 \%$ and her supine BP fell to $98 / 60 \mathrm{mmHg}$. At this time, her intravenous fluids were increased; she was given albumin and started on dexamethasone $8 \mathrm{mg}$ stat and $4 \mathrm{mg}$ intravenously, every six hours.

By the following day, her condition had improved. The diarrhoea diminished to just about two bowel actions per day with no blood but both the megacolon and abdominal pain persisted. By the fourth day of post fecal transplantation, the patient was on her feet and having a soft diet. She reported no further blood in the stools which were pasty by then. By day six, however, her diarrhoea returned and she reported up to six motions per day. Two subsequent courses of bacteriotherapy were undertaken - this time the mode of delivery was in the form of fecal retention enemas using the same donor (10). After her second enema, the patient was still having two to three bowel actions per day along with postevacuation cramps. There was no further bleeding, and her appetite was improving.

The following week, another fecal transplant was done. On that occasion, the route of delivery was via the duodenum. The patient was sedated and a 'through the scope' guide-wire (Wiltex Corp) was placed into the second part of the duodenum, the scope withdrawn and a size 14 nasogastric tube placed via the wire-guide, into the second part of the duodenum. The emulsion was delivered through the tube without complication. There was marked improvement three days following the procedure. The patient was fully ambulant and had formed stools, and her appetite returned. She was discharged with a one week appointment for review. 


\section{DISCUSSION}

Fecal bacteriotherapy is an accepted but not commonly performed treatment for recurrent and/or antibiotic-resistant Clostridium difficile infection and interest in this form of treatment for IBD is increasing rapidly. Worldwide, roughly 450 cases of fecal trans-plantation for treatment of $C$ difficile infection have been reported $(11,12)$. This report is arguably the first in the use of this procedure in the English-speaking Caribbean.

This case should serve to remind the reader of the following four pertinent points:

* Clostridium difficile infection can often complicate IBD - in this case Crohn's colitis (8)

* the usefulness of this therapy in refractory and fulminant Clostridium difficile infection unresponsive to 48 hours of conventional therapy $(11,12)$

* that the presence of IBD often masks the classical morphologic features of pseudomembrane formation in Clostridium difficile infection (8), and finally

* to be aware of the potential presence of Clostridium difficile infection in the Caribbean community

Intestinal microbiota transplantation involves the transfer of stool from a healthy donor to the colon of a sick recipient in an effort to reverse the recipient's colonic pathology. Veterinarians have been using this procedure (transfaunation) to treat animals for a variety of gut disorders. $C$ difficile, although first isolated by Hall and O'Toole in 1935, was not implicated as a cause of pseudomembraneous colitis until 1978 (13). The procedure was first used in humans in 1958. Intestinal microbiota transplantation has also been used to treat IBD and irritable bowel syndrome (IBS) as both are thought to be causally related to intestinal microbiota (7).

Pathogenic $C$ difficile strains produce exotoxins enterotoxin $\mathrm{A}$ and cytotoxin $\mathrm{B}$ - which damage the cytoskeleton of the enterocyte by mechanisms that involve the release of leukotrines and cytokines (1). Between 2003 and 2006, more frequently occurring infections were being reported in Canada, the USA and Europe. It is the major cause of infectious diarrhoea in Dublin, Ireland. As in our patient, the classical findings of pseudomembrane formation is not usually seen when Clostridium difficile infection occurs alongside IBD. Clostridium difficile infection worsens IBD outcome. Patients with combined Clostridium difficile infection and IBD are six times more likely to have surgery, and have a four times higher mortality than patients with either Clostridium difficile infection or IBD alone (14).

A meta-analysis of 317 patients across 27 case series and reports supports intestinal microbiota transplantation as a highly effective form of treatment for Clostridium difficile infection when standard therapies have failed (7). Complete resolution occurred in $92 \%$ of cases $-89 \%$ after a single treatment - with relapses being uncommon. Although it is not fully known why this form of treatment works, the weight of evidence points to the fact that intestinal microbiota transplantation restores the composition and function of the intestinal microbiome. Other alternatives to standard therapy eg probiotics, toxin-binding molecules, immunoglobulins and $C$ difficile vaccination, have not yet proven to be of value (7).

\section{Addendum}

The patient defaulted on her follow-up appointment two weeks post discharge. She was seen by another physician and steroids continued but she stopped on her own and was readmitted four weeks later in an Addisonian crisis and succumbed.

\section{ACKNOWLEDGEMENT}

The author wishes to thank Nurse Melissa Modeste-Alexis, senior endoscopy nurse who assisted in all procedures in respect of this patient and prepared all donor specimens for transplantation.

\section{REFERENCES}

1. Hookman P, Barkin J. Clostridium difficile associated infection, diarrhea and colitis. World J Gastroenterol 2009; 15: 1554-80.

2. Yearsley K, Gilby L, Ramdass A. Proton pump inhibitor therapy is a risk factor for Clostridium difficile-associated diarrhea. Aliment Pharmacol Ther 2006; 24: 613-9.

3. Rouphael N, O'donnell J, Bhatnagar J, Lewis F, Polgreen P, Beekmann S. Clostridium difficile-associated diarrhea: an emerging threat to pregnant women. Am J Obstet Gynecol 2008; 198: 635.

4. Zerey M, Paton B, Lincourt A, Gersin K, Kercher K, Heniford B. The burden of Clostridium difficile in surgical patients in the United States. Surgical Infect (Larchmt) 2007; 8: 557-66.

5. Dupont $H$. The search for effective treatment of clostridium difficile infection. New Engl J Med 2011; 364: 473-5.

6. Mcfarland L, Elmer G, Surawicz C. Breaking the cycle: treatment strategies for 163 cases of recurrent Clostridium difficile disease. Am J Gastroenterol 2002; 97: 1769-75.

7. Gough E, Shaikh H, Manges A. Systematic review of intestinal microbiota transplantation (fecal bacteriotherapy) for recurrent Clostridium difficile infection. Clin Infect Dis 2011; 53: 994-1002.

8. Goodhand J, Alazawi W, Rampton D. Systematic review: Clostridium difficile and inflammatory bowel disease. Aliment Pharmacol Ther 2011; 33: 428-41.

9. Johnson D. Fecal transplantation for C difficile: an evolving "art" [Internet]. 2012 [cited 2012 Nov]. Available from: http://www. medscape.com/viewarticle $/ 765243$ ? $\mathrm{src}=\mathrm{mp} \&$ spon $=38$

10. Damman C, Miller S, Surawicz C, Zisman T. The microbiome and inflammatory bowel disease: is there a therapeutic role for fecal microbiota transplantation? Am J Gastroenterol 2012; 107: 1452-9. DOI: 10.1038/ajg.2012.79.

11. Brandt L. Fecal transplantation for the treatment of Clostridium difficile infection. Gastroenterol Hepatol 2012; 8: 191-4.

12. Brandt L. Fecal microbiota transplant. Gastrointestinal Nursing 2012; 10: 6-7.

13. George W, Volpicelli N, Stiner D, Richman D, Liechty E, Mok H et al. Relapse of pseudomembranous colitis after vancomycin therapy. New Engl J Med 1979; 301: 414-5.

14. Sinh P, Barrett T, Yun L. Clostridium difficile infection and inflammatory bowel disease: a review. Gastroenterol Res Pract 2011; 2011: 136064. DOI: 10.1155/2011/136064. Epub 2011 Sep 12. 